\title{
Brokering in Electronic Insurance Markets
}

\author{
Luís Nogueira $^{1}$, Eugénio Oliveira ${ }^{2}$ \\ 1 Instituto Superior de Engenharia do Porto, \\ Instituto Politécnico do Porto \\ luis@dei.isep.ipp.pt \\ 2 Faculdade de Engenharia, \\ Universidade do Porto, LIACC \\ eco@fe.up.pt
}

\begin{abstract}
The insurance industry appears to be lagging in the rapid evolution of financial services an e-commerce, as it faces strategic challenges in utilising the Web. The majority of insurers continue to build sites that are structured on a corporate orientation rather than customer needs. Customers are left on their own to figure out where they should find useful information. In this paper we propose an agent-mediated insurance brokering system using a flexible negotiation model that includes multi-attribute bidding as well as some kind of learning capabilities. Moreover, in the core of the provided brokering facility, we are using conceptual clustering procedures as an approach to better match customers and insurance product offers, providing a valuable add-on to both customer's and sellers' sides. Particular attention is paid to the interpretation of the generated communities. For this purpose, we use a metric to identify the representative insurance product configuration of each community. To improve broker's evaluation of received insurer's bids we propose the automatic construction of the customer's profile, reflecting its preferences on all the attributes of an insurance product.
\end{abstract}

\section{Introduction}

Despite e-commerce's huge impact on business in general, the insurance industry has yet to fully embrace it. The insurance industry faces several obstacles to e-commerce, including customer attitudes, complex insurance policies, state regulations and the traditional agency distribution system. However, with the rapid growth of information technology infrastructures, online insurance offers immense opportunities for insurance companies.

The success of the sale of an insurance policy depends on how good the requirements of the insurer have been matched with the terms of the policy. In the conventional insurance industry, the insurance company initially informs its customers through advertisements. Advertisements are made either through passive channels like newspapers, magazines, billboards, radio and television, or through active channels like human insurance agents. E-insurance employs the Internet to reach customers through advertisements more effectively since it integrates the traditional passive and active channels of advertisement into 
one. Advertisement banners, e-mail notifications and coupons are used to replace passive media, while software agents can replace their active human counterparts.

However, it's a much difficult task to match the insurance requirements of a customer with appropriate financial complex products than, for example, finding those shops that sell a specific book. An insurance policy has benefits, conditions and exclusions that add detail to the high level coverage features advertised by the insurer.

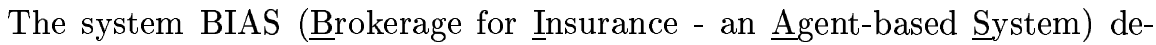
scribed in this paper presents a new approach to insurance products brokering and has the potential to improve the quality of customer service by ensuring that individual customer needs are reflected in the products offered.

\section{The Current State of Online Insurance}

Despite the increasingly rapid advances made in computer technology, companies in the insurance industry are making the most use of the new technical possibilities offered today in their internal operations only, while hardly employing them at all when negotiating with their customers. A recent comparative study of 25 web-based Internet sites offering comparative term life insurance information from Consumer Federation of America has showed us that not all sites are useful for getting quotes. Some of them are too difficult to use and others are little more than referral services where the customer is put in touch with a human agent, something that customers, most of the time, do not need the Internet to achieve. Several of the exiting quote services do not include no commission insurance companies, because many of this sites make money through commissions on sales and do not show this companies in their service since it would reduce their incomes [5].

Most web sites offering online quotation and purchase of insurance products are implemented by insurers and sell directly to the customer, excluding the broker. Brokers, however, provide a valuable service and are widely used by customers. Yet, online brokerage is rare.

Those sites, which do offer a brokerage service, do so by drastically simplifying the problem: they standardise the products which insurers are permitted to offer through the site. The broker's role is then reduced to collect a standard set of information from the customer and negotiate standard coverages. This gives no advantage for any of the players because customers are provided with a more limited choice of products, which may not meet all their requirements, insurers have limited flexibility in product design, targeting and pricing, and brokers lose their traditional role.

\section{Agent Mediated Insurance Brokerage}

\subsection{Our Proposal}

It is our belief that intelligent agents are well suited to deal with the insurance brokering problem in a distributed manner. By configuring a society of intelligent 
agents, each one charged with autonomously carrying out different specific functionalities, the insurance broker system will not only be able to analyse products being offered, but will also deduce useful information regarding the current state of the market.

In [7] we have presented a distributed, intelligent agent-based system, making it possible the electronic commerce of insurance products. Our approach for an agent-based insurance products assisted electronic market includes an agent representing each of the insurers, an agent representing the customer and a broker agent for intelligent brokering services. Each insurer has full ownership of its agent, ensuring that all strategic information remains confidential. The goal is to support distribution of a full range of insurance products from several different insurers without the need to modify or constrain them in standard rigid formats for electronic commerce purposes.

In the core of the provided brokering facility, we are using conceptual clustering procedures as an approach to better match customers' requirements and insurance product offers, providing a valuable add-on to both customers' and insurers' sides. Our insurance brokering system is then using COBWEB as a tool for grouping potential customers in meaningful classes we call communities. The question is whether there is any meaning in the generated communities, that is, if they associate users with a limited set of common interests. For this reason we use a metric to decide which preferences are most representative for each community. We try to construct a prototypical model, that we call stereotype, for each community, which is representative of its users and significantly different from other communities of users. This approach allows the insurance company to target product configurations at specific market segments, and avoids the need to ask all customers the same typically large number of product specific questions.

In order to reach an agreement about a particular insurance product a negotiation process is started by the broker. This negotiation process comprises several rounds, starting when the broker sends and announcement for all the insurer agents in the market. We have adapted the Q-Negotiation algorithm [11][10] to the insurance brokering problem. This algorithm uses a reinforcement learning strategy based in Q-learning for the formulation of new proposals. The broker negotiates an insurance product configuration with insurers, trying to fulfil the specific needs of the customer. This configuration, defined by the customer, includes several, possible dependent, components. At each round, bids' evaluation is done through a multi-issue function that encodes the customer's preferences.

The broker helps the insurer agents on their task of formulating new proposals by giving them some hints about the direction they should follow in their negotiation space. The response to proposed insurance configurations is formulated by the broker as a qualitative feedback, which reflects the distance between the values indicated in a specific proposal and the best one received so far. Insurer agents will use this feedback information to its past proposals, in order to formulate, in the next rounds, new proposals trying to approach the customer's 
preferences. The negotiation ends when a deadline is reached or a satisfactory proposal is received.

A customer agent coordinates the dialogue between the customer and the broker, passing on information as appropriate. Besides learning the customer profile, it offers the customer a flexible navigation tool that allows the exploration of the received proposals. This is particularly useful because the customer wish to express product feature preferences and view the corresponding proximity of each offered product. The result is a ranking of products, which can be tuned by the customer by varying the preferences and viewing the consequent effect on the ranked list [12]. Such a navigation tool encourages the user to consider non-price related features and helps the customer to explore the trade-off between product features and price. This is not just of benefit to the customer, because insurers have also the means of drawing attention to their products' distinguishing features other than price [4]. This helps the customer to make an informed purchase decision.

\subsection{Phases of the Brokering Problem}

Our model for the insurance brokering activities divides the interaction process aiming at solving the problem into the phases represented in figure 1. Besides relevant services like negotiation protocols and ontology-based services, the Broker Agent provides a facility to build up, memorise and exploit customer's stereotypes. Although this concept will be elaborated later on section 5, the reader can see a stereotype as a classification of a set of features and characteristics associated with a specific sub-set of customers.

In phase 1, the user sends, trough its Customer Agent (CA), his personal characteristics and needs. The Broker Agent (BA), in phase 2, replies with the stereotype of the customer's community, indicating the representative preferences of its users. Based on this received stereotype the user defines the allowed range for the insurance product attributes' values. This definition includes attaching a degree of importance (weight value) for each one of the product's attributes (in a range from low to high) and the increasing order of preference for the attributes' values. The CA sends this request to the BA (phase 3).

In phase 4, the BA sends an announcement to each Insurer Agent (IA), starting a negotiation process. Each IA replies with bids to BA, which are then evaluated according to customer preferences, extracting relevant features from this bids (phase 5). This bids, plus relevant information, is sent to CA (phase 6). CA offers the customer a flexible navigation tool that allows the exploration of the received proposals.

The customer has now the opportunity to define a counter-proposal and select the insurers he wishes to continue to negotiate (phase 7). The response to proposed bids (phase 8) is formulated by the Broker Agent as a qualitative feedback, which reflects the distance between the values indicated in a specific proposal an the optimal one received so far, which can be classified in one of three categories: sufficient, bad or very_bad. The reason why the BA compares a particular proposal with, not the optimal values for the customer, but the best 


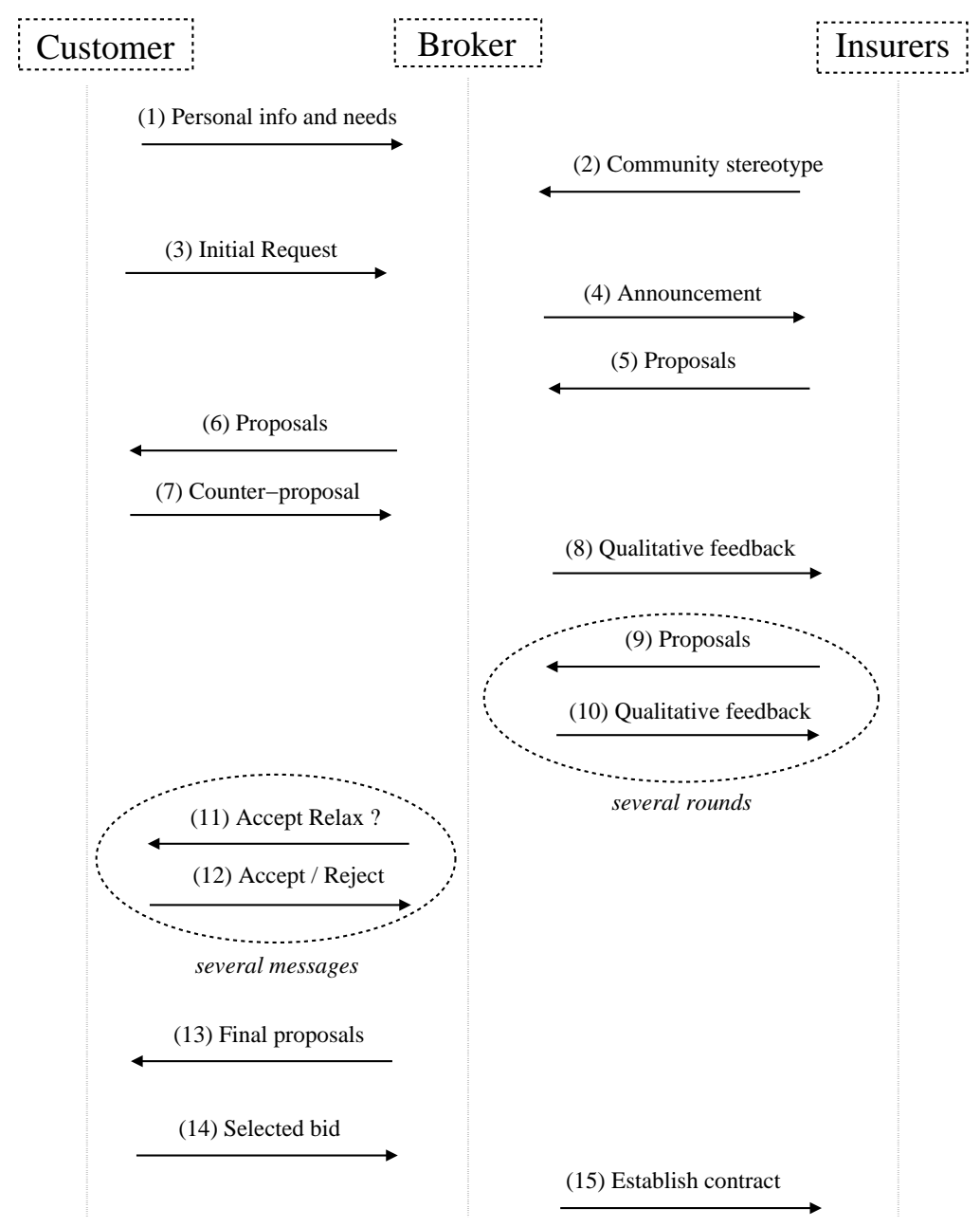

Fig. 1. Phases of the brokering problem

one received so far can be explained by the fact that it's more convincing to say to an Insurer Agent that there is a better proposal in the market than saying that its proposal is not the optimal one.

At each negotiation round bids are evaluated and the one with the greatest evaluation value is considered the winner (phase 9 and 10). When this negotiation process ends, BA starts a new interaction with CA. This interaction will ultimately direct the system to a suitable solution through a constraint satisfaction process (phase 11 and 12). This conversation takes the form of a sequence 
of questions whose aim is to reduce alternatives rather than simply sort them. This dialogue is detailed in [7].

If this conversation has produced a valid number of alternatives, BA initiates phase 13 by ranking selected proposals according to the user utility function results, and send them plus relevant information to CA. The user either rejects or agrees with one of the received proposals (phase 14).

If the user has selected one of the proposed insurance products, BA starts phase 15 , establishing a contract with the winning IA.

\section{Learning Customer Communities}

Customer communities can be constructed automatically using an unsupervised learning method. The COBWEB algorithm [1], that we use in this work, performs conceptual clustering. Conceptual clustering is a type of learning by observation that has as its goal the unsupervised classification of a set of objects. Classification is unsupervised in the sense that there are no a priori target classes used during training.

COBWEB is an incremental conceptual clustering algorithm that represents concepts probabilistically. The term incremental means that objects are incorporated into the concept structure as they are observed. An object is a vector of feature-value pairs. In our system, objects are user's characteristics. COBWEB is designed to produce a hierarchical classification scheme. It carries out a hill-climbing search through a space of schemes, and this search is guided by an heuristic measure called category utility [3].

The category utility of a partition is measured by the following equation:

$$
C U=\frac{\sum_{k}\left(P\left(C_{k}\right)\left[\sum_{i} \sum_{j} P\left(A_{i}=V_{i j} \mid C_{k}\right)^{2}-\sum \sum P\left(A_{i}=V_{i j}\right)^{2}\right]\right)}{k} .
$$

where $k$ is the number of categories or classes, $C_{k}$ is a particular class, $A_{i}$ refers to one of the $I$ attributes and $V_{i j}$ is one of the $J$ values for attribute $A_{i}$.

COBWEB incorporates objects into the concept hierarchy using four clustering operators: placing the object in an existing cluster, creating a new cluster, combining two clusters into a new one (merging) and dividing a cluster (splitting). Given a new object, the algorithm applies each of the previous operators and selects the hierarchy that maximises category utility.

\section{Learning Communities Stereotypes}

Our insurance brokering system is then using COBWEB as a tool for grouping potential customers in meaningful classes we call communities. But, besides the customer data used for incrementally build up customer's communities, a customer is characterised by its own preferences and by the insurance configuration chosen in the negotiation process. In order to help customers defining their needs, 
the broker must associate a "typical" insurance configuration to each constructed community. Thus, the natural way to define meaningful stereotypes associated to customer's communities is by trying to identify patterns that are representative of the participating users' preferences. We try to construct a prototypical model for each community, which is representative of its users and significantly different from other communities of users.

Our system is using a metric to measure the increase in the frequency of a specific preference or negotiation result within a given community, as compared to the default frequency in the whole number of available observations [9]. In [8] and [2] the increase in frequency was used as an indication of the increase in the predictability of a feature (a given preference, for example) within the community. Given a component $c$ (a user preference), with the default frequency $f_{c}$, if the frequency of this component within a community $i$ is $f_{i}$, the frequency increase is defined as a simple difference of the squares of the two frequencies:

$$
F I_{c}=f_{i}^{2}-f_{c}^{2} .
$$

When $F I_{c}$ is negative there is a decrease in frequency and the corresponding component is not representative of the community. A community's representative characteristic is found through $F I_{c}>\alpha$, where $\alpha$ is pre-established as the required threshold for considering that frequency increase enough relevant.

\section{Learning Customer Profiles}

The customer approaches the broker with a requirement: he wish to buy an insurance policy to cover certain risks. This requirement will usually be incomplete and uncertain, and the customer will not be aware of all the options available. As such, if the broker's evaluation of the received proposals were only based on the attributes that the customer is able to specify the result might be very poor. We propose the construction of a user profile, induced from the set of selected proposals for the next negotiation rounds. This profile reflects the customer's preferences on all the attributes of an insurance product. The training set consists of the proposals that the user found interesting.

The task of constructing the customer profile involves judging whether an attribute of an insurance product is relevant or irrelevant for the user. It would be very frustrating and time consuming for a user to interact with an agent that starts with no knowledge but must obtain a set of positive and negative examples from user feedback. To reduce user evaluation burden, BIAS considers only examples that are interesting for the user (only positive examples).

Formally, a proposal is described as a vector $P=\left(a t r_{1}, a t r_{2}, \ldots, a t r_{n}\right)$ of $n$ attributes. The attributes can have binary, nominal or numerical values and are derived from the system ontology. The task of the learning method is to select the desired insurance configuration, based on a training set of $m$ input vectors, that is, the selected proposals for the next negotiation rounds.

The construction of the customer profile is based on the correlation between the content of the selected proposals and the user's preferences as opposed to 
methods based on the correlation between customers with similar preferences. This preferences can be determined by using either explicit or implicit feedback. Explicit feedback requires the user to evaluate received proposals on a scale. In implicit feedback the user's preferences are inferred by observing user's actions, which is more convenient for the user. That's why we have adopted it in BIAS, despite the greater complexity in implementation.

In BIAS, insurer's proposals are represented as an $n$-dimensional vector, where each dimension corresponds to a distinct attribute and $n$ is the total number of possible attributes. It's possible to extract a vector $V_{i}$ from each proposal, $V_{i}=\left[x_{1}: F p_{1}, x_{2}: F p_{2}, \ldots, x_{n}: F p_{n}\right]$, where $F p_{i}$ indicates how frequently an attribute-value pair $x_{i}$ appears in a particular proposal. If the proposal does not contain $x_{i}$ then $F p_{i}$ is zero.

Customer profiles can be represented just like documents by one or more vectors. The profile is represented as an $n$-dimensional vector, where $n$ is the total number of possible attributes for an insurance product. Each dimension is represented by $m$ vectors, where $m$ is the total number of possible values for that attribute. In this approach a weight $w_{i}$ is assigned to each value, based on how often the attribute-value pair appears in a particular proposal, $F p_{i}$, and how frequently is occurs in the selected set of proposals, $F s_{i}$.

$$
\begin{aligned}
& F s_{i}=\sum_{j=0}^{n} F p_{i j} \\
& w_{i}=F p_{i} * \frac{F s_{i}}{n}
\end{aligned}
$$

We propose the algorithm 1 for the construction of the customer profile. The assumption behind this algorithm is that the more times a value appears in the selected set of proposals, the more relevant it is for the user.

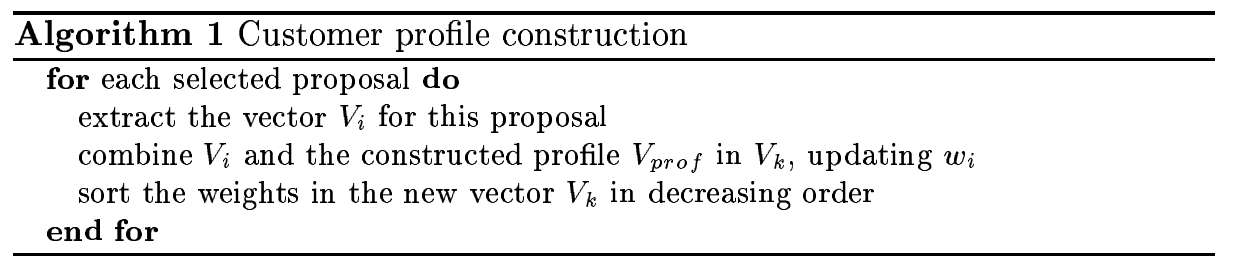

This algorithm runs whenever a user selects a proposal for the next negotiation rounds. Thus, the customer profile is incrementally and continuously updated.

\section{$7 \quad$ Results}

In [6] we have showed the importance of the generated stereotypes for each specific customer. The Broker Agent, through the construction of customer's 
communities and the identification of the representative preferences of its users, is able to provide needed help and advise in defining customer's needs, selecting a policy and dealing with insurers.

The quality of the generated descriptions for each community increases as we go down in the hierarchy constructed by COBWEB. Communities in lower levels are more concise and the extracted model has greater significance. The tests have showed us that the insurance attributes that are chosen by either too few or too many users do not appear in the constructed community stereotype. In the former case the algorithm ignores them during learning and in the latter case, they correspond to such general interests, that they cannot be attributed to particular communities. Filtering out this two types of preferences is a positive feature of the used metric [9], greatly improving the quality of the generated stereotypes.

Changes in electronic insurance commerce, introduced in BIAS, are of benefit of both customers and insurers. By increasing the degree and the sophistication of the automation process, commerce becomes much more dynamic, personalised and context sensitive. From the customers' perspective, it is desirable to have software that could search all the available offers to find the most suitable one and then go forward through the process of actually purchasing the product. From the insurer's perspective it is desirable to have software that could vary its own offering depending on the customer it is dealing with, on what its competitors are doing and the current state of its own business.

\section{Conclusions}

In most current e-commerce applications, the buyers are generally humans who typically browse through a catalogue of well defined products (books, computer components, CDs) and make fixed price purchases. However, there are important differences between selling this type of goods and selling insurance and other financial products over the Internet. This paper explores some of those differences from both the insurers' and customers' perspectives and describes the development of a multi-agent system through which products (and services) offered by insurance companies could be better evaluated and selected. From the customers side point of view, more interesting information can be found, even things that the customer did not think of before. On the other hand, the BIAS system, with its intimate knowledge of who the user is and what he wants, can shorten the time needed for finding an appropriate insurance product. Insurers can then use information automatically collected during negotiation to develop a more customer-directed kind of marketing strategy. Information about the customer can be used to find out what he is interested in and, therefore, a more personalised product could be offered.

Different communities of users can be identified and used to improve the exploitation of an insurance brokering service. The construction of those communities is achieved using an unsupervised learning technique. We also use a 
specific metric to decide which are the representative preferences of a user's community.

We propose the construction of a user profile, induced from the set of selected proposals for the next negotiation rounds. This profile reflects the customer's preferences on all the attributes of an insurance product.

We have also adapted an advanced negotiation protocol, suitable for multiissue negotiation in electronic commerce activity. A learning capability was also included enabling agents to become more effective in a dynamic market by learning with past experience through the qualitative feedback received from their opponents.

\section{References}

1. D. H. Fisher. Knowledge acquisition via incremental conceptual clustering. $M a$ chine Learning, 2:139-172, 1987.

2. J.B. Weinberg G. Biswas and D. Fisher. Iterate: A conceptual clustering algorithm for data mining. IEEE Transactions on Systems, Man and Cybernetics, 28:100-111, 1998.

3. M.A. Gluck and J.E. Corter. Information, uncertainty and the utility of categories. In Proceedings of the 7th Conference of the Cognitive Science Society, pages 283287, 1985.

4. R. Guttman, A. Moukas, and P. Maes. Agent-mediated electronic commerce: A survey. Knowledge Engineering Review, 13(2):147-159, 1998.

5. J. Robert Hunter and James H. Hunt. Term Life Insurance on the Internet: An Evaluation on On-line Quotes. Consumer Federation of America, 2001.

6. Luís Nogueira. Sistema multi-agente para mediação electrónica de seguros. Technical report, LIACC, University of Porto, 2002.

7. Luís Nogueira and Eugénio Oliveira. A multi-agent system for e-insurance brokering. In R. Kowalczyk, J. Müller, H. Tianfield, and R. Unland, editors, to appear in Agent Technologies, Infrastructures, Tools, and Applications for e-Services, volume 2592 of Lecture Notes in Artificial Intelligence. Springer-Verlag, 2003.

8. G. Paliouras, V. Karkaletsis, C. Papatheodorou, and C. Spyropoulos. Exploiting learning techniques for the acquisition of user stereotypes and communities. In Proceedings of the International Conference on User Modelling (UM '99)., 1999.

9. G. Paliouras, C. Papatheodorou, V. Kakaletsis, C. Spryropoulos, and V. Malaveta. Learning user communities for improving the services of information providers. Lecture Notes in Computer Science, 1513:367-384, 1998.

10. Ana Paula Rocha and Eugénio Oliveira. Adaptative multi-issue negotiation protocol for electronic commerce. In Proceedings of The Fifth International Conference on The Practical Application of Intelligent Agents and Multi-Agent Technology (PAAM 2000),, Manchester, UK, April 2000.

11. Ana Paula Rocha and Eugénio Oliveira. Agents advanced features for negotiation in electronic commerce and virtual organisations formation process. Lecture Notes in Computer Science, 1991:77-96, 2001.

12. M. Stolze. Soft navigation in electronic product catalogs. International Journal in Digital Libraries, 3(1):60-66, 2000. 